\title{
DECP: A Distributed Election Clustering Protocol for Heterogeneous Wireless Sensor Networks
}

\author{
Xianghui Wang and Guoyin Zhang \\ College of Computer Science and Technology, Harbin Engineering University, 150001 \\ Harbin, China \\ Wangxianghui@hrbeu.edu.cn
}

\begin{abstract}
Heterogeneous wireless sensor networks are energy limited and imbalance networks, load balancing and energy efficiency is the most challenging task in these networks. In this paper, we propose a distributed election clustering protocol to prolong the stable region of wireless sensor networks, which is based on remaining energy and communication cost to elect suitable cluster-head nodes. Compared with classical clustering protocol, our protocol can maintain load balancing of networks, and extremely prolong the stable region of network lifetime.
\end{abstract}

Keywords: wireless sensor networks, cluster, heterogeneous.

\section{Introduction}

Wireless sensor networks have been envisioned to have a wide range of application in both military and civilian domains [1]. Due to the limitation of sensor node energy, researchers have designed lots of energy-efficient routing protocols to prolong the lifetime of sensor networks. These protocols are mainly for homogeneous sensor networks, and hardly used in heterogeneous environments. In this paper, we propose DECP, a new distributed election clustering protocol for tow-level heterogeneous wireless sensor networks. DECP is heterogeneous-aware, in the sense that election probabilities are weighted by residual energy and communication cost. When energy is imbalance in local area, high energy node is prior to be the cluster head, and when energy is balance, communication cost is considered first. This election mechanism prolong the time interval before the death of the first node (stable region), which is crucial for many applications where the feedback from the sensor network must be reliable. We show by simulation that DECP could implement load balancing and provides long stable region than the classical protocols LEACH [2] and SEP [3].

\section{Clustering Parameters}

In order to prolong the stable region, DECP attempts to maintain the constraint of well balanced energy consumption. High energy nodes should have more opportunity to 
become cluster-head, and low energy nodes should not be the cluster-head until there are no high energy nodes within their detective range. We use Average Power Distinction (APD) to evaluate the power level of node $i$.

$$
A P D_{i}=1-\frac{\sum_{j \in N B R_{i}} E_{j}^{\text {current }}}{\left|N B R_{i}\right| \cdot E_{i}^{\text {current }}}
$$

$N B R_{i}$ is the set of neighbors of node $i$, which are located in the detective range of $\operatorname{nod} i$, and $\left|N B R_{i}\right|$ is the total number of nodes in $N B R_{i} . E_{i}^{\text {current }}$ is the current residual energy of node $i$.

$A P D_{i}$ reflects the power distinction between the node $i$ and its neighbors. If $A P D_{i}$ is more than zero, node $i$ is a high energy node, which means that node $i$ have more energy than his neighbors; if $A P D_{i}$ is less than zero, node $i$ is a low energy node, and should have less opportunity to be the cluster-head node.

In heterogeneous networks, we must both consider the average power distinction and communication cost for cluster-head selection, so we use $m$ Cost to evaluate communication cost in heterogeneous networks.

$$
m \operatorname{Cost}_{i}=\left(1-A P D_{i}\right) \cdot \frac{\sum_{j \in N B R_{i}} d_{i, j}^{2}}{\left|N B R_{i}\right|}=\frac{\sum_{j \in N B R_{i}} E_{j}^{\text {current }} \cdot \sum_{j \in N B R_{i}} d_{i, j}^{2}}{\left|N B R_{i}\right|^{2} \cdot E_{i}^{\text {current }}}
$$

$d_{i, j}$ is the distance between node $i$ and node $j$, and this value should be computed by receiving sensitivity. $m$ Cost provides a unified criterion for all nodes to select cluster-head nodes, which means that all nodes could use mCost to select cluster-head nodes, which is the nodes with high energy and low communication cost.

\section{DECP Protocol}

A clustered sensor network is partitioned to a number of clusters. Node $i$ working as a cluster-head node is denoted by $c h_{i}$. The set of all cluster-head nodes is denoted by $\mathrm{CH}, \mathrm{CH} \subseteq N$, where $\mathrm{N}$ is the set of all nodes including cluster-head nodes and non-cluster-head nodes. Now we describe DECP protocol in detail.

In clustering process, all the nodes broadcast its current energy information Energy_msg, and then overhear the energy message from the other nodes. When nodes have sufficient information about its neighbors, such as distance and current energy, nodes calculate $\boldsymbol{m}$ Cost about itself and broadcast $\boldsymbol{m}$ Cost to its neighbors. According to $\boldsymbol{m}$ Cost , each node selects the candidate node which has the minimal $\boldsymbol{m}$ Cost , and sends Vote_msg to the candidate node. The nodes which receive the most Vote_msg in neighbors, will announce that the cluster-head nodes are elected, and all non-cluster-head nodes chose one nearest cluster-head to join the cluster. 
Table 1. DECP protocol

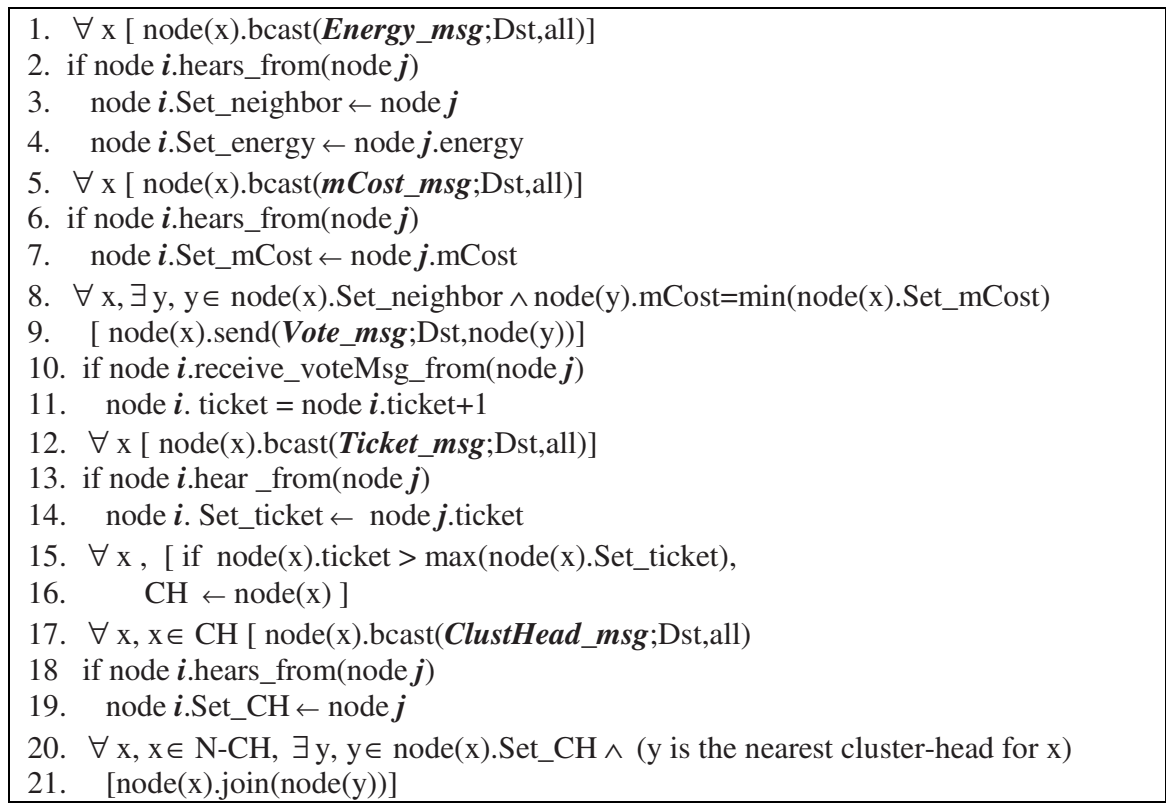

\section{Simulation and Evaluation}

We simulate a wireless network of 100 nodes in a $100 \times 100$ square area using MATLAB, and the sink node is located in the center of the area. We assume that $m$ is the percentage of the nodes which are equipped with $a$ times more energy than the normal nodes. The initial energy of normal node is $0.1 \mathrm{~J}$, so the initial energy of advance node is $0.5(a+1) \mathrm{J}$. In order to measure the energy consumption for data transmission, we used the same energy consumption model introduced in [4], using radio electronics energy $E_{\text {elec }}=50 \mathrm{nj} / \mathrm{bit}$, radio amplifier $\varepsilon_{f s}=10 \mathrm{pj} / \mathrm{bit} / \mathrm{m}^{2}$, the consumption of data fusion $E_{\text {fusion }}=5 \mathrm{nj} / \mathrm{b}$ it/ $/$ message, 2000 bit-size sensed data package and 50 byte-size broadcast package.

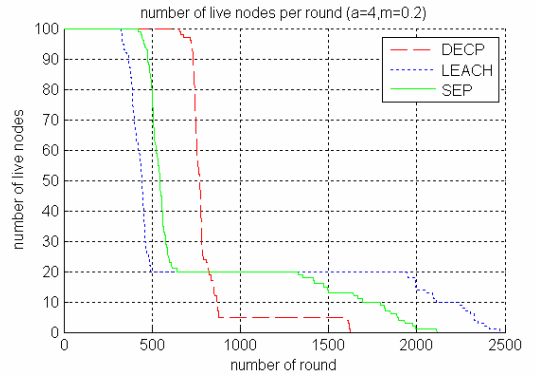

Fig. 1. Number of live nodes

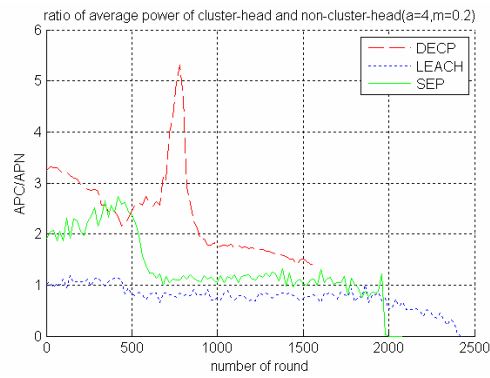

Fig. 2. Contrast of APC/APN 
We compare the performance of DECP protocol with LEACH and SEP in the same heterogeneous setting, where $m=0.2$ and $a=4$.

Figure1 shows that DECP extremely extend the stable region compared to LEACH by $102.16 \%$ and SEP by $49.17 \%$. On the other hand, DECP increase the ratio of stable region in network lifetime. In DECP, the ratio is $40.31 \%$, in LEACH and SEP it is $13.11 \%$ and $19.91 \%$. DECP select high energy nodes to be the cluster-head for load balancing, and low energy nodes spend less energy than high energy nodes. So DECP avoid the death of low energy nodes too earlier and prolong the stable region of the wireless sensor networks.

Figure 2 shows that the APC/APN of DECP is higher than LEACH and SEP. APC/APN is the ratio of average power of cluster-head nodes and non-cluster-head nodes. If APC/APN more than one, it means that the cluster-head nodes have more average energy than non-cluster-head nodes; if APC/APN is equal one, it means that the cluster-head nodes have the same average energy as non-cluster-head nodes. LEACH use random arithmetic to select cluster-head nodes, so APC/APN is approximately equal one. In SEP, the advance nodes have more opportunity to be the cluster-head, but the arithmetic still use random mechanism to select cluster-head, so the APC/APN is just more than one. DECP has the best performance in APC/APN, because DECP do not use random mechanism for cluster-head selecting, thus DECP could accurately select the high energy node with low communication cost to be the cluster-head, and implement load balancing.

We also analyze the performance of DECP with different $m$ and $a$, the experiment result show that the stable region of DECP is far more than that of LEACH and SEP, even in the homogeneous networks $(a=0)$.

\section{Conclusion}

In this paper, we present DECP, a distributed election clustering protocol for tow-level heterogeneous wireless sensor networks. Our protocol does not need any of global energy knowledge at clustering process. As long as nodes exchange local information, cluster-head nodes could be selected. Furthermore, DECP is scalable as it dose not required any of exact position of each node in the filed. Our protocol use local energy information and communication cost for clustering, it is prolong the stable stage of the wireless sensor networks.

\section{References}

1. I.F Akyildiz, W. Su, Y. Sankarasubramaniam, E. Cayirci: A survey on sensor networks. IEEE Communications Magazine (2002) 102-114

2. W.R. Heinzelman, A.P. Chandrakasan, H. Balakrishnan: Energyefficient communication protocol for wireless microsensor networks. In Proceedings of the 33rd Hawaii International Conference on System Sciences (2000)

3. Georgios Smaragdakis, Ibrahim Matta, Azer Bestavros: SEP: A Stable Election Protocol for clustered heterogeneous wireless sensor networks. IEEE (2003)

4. W. Heinzelman, A. Chandrakasan, H. Balakrishnan: An Application-Specific Protocol Architecture for Wireless Microsensor Networks. IEEE Trans, Wireless Comm(2002) 660- 670 